\title{
Wedge-cell technique as a simple and effective method for chromatic dispersion determination of liquid crystals
}

\author{
Katarzyna A. Rutkowska, Kamil Orzechowski, and Marek Sierakowski \\ ${ }^{I}$ Faculty of Physics, Warsaw University of Technology, Koszykowa 75, 00-662 Warszawa,
}

Received June 27, 2016; accepted June 29, 2016; published June 30, 2016

\begin{abstract}
In this letter we present a wedge-cell method and then compare it with other techniques that can be used to determinate refractive indices of liquid crystalline materials. This simple goniometric technique is of particular significance when used for liquid crystalline materials characterized by high refractive indices, for which refractometric methods are approaching their upper limits. Importantly, the measurement technique proposed here requires a relatively smal amount of liquid crystalline material and gives appropriate results for different light sources from the wide spectral range, allowing thus for chromatic dispersion curves (in different temperatures) to be obtained.
\end{abstract}

Liquid crystals (LCs) are unique anisotropic materials with special optical properties. In particular, their refractive indices strongly depend on temperature and molecular orientation, which plays an important role in practical applications of LCs in photonics. Most of LCs are uniaxial and their optical birefringence, defined as a difference between an extraordinary and ordinary refractive index $\left(\Delta n=n_{e}-n_{o}\right)$, together with their chromatic dispersion, are the most important parameters to be determined in order to ensure proper design, fabrication and functionality of LC-based photonic elements and devices. It is critical thus to deliver and elaborate a convenient and accurate method for refractive index measurements, specifically in a broad spectral range and as a function of temperature [1-7]. Typically, optical characterization of liquid crystalline materials in terms of chromatic dispersion is performed at room temperature, while the thermal dependence of refractive indices is usually given for one wavelength only (e.g. $589 \mathrm{~nm}$ which is characteristic for D-sodium-line). Such information is insufficient when needed for proper design and operation of LC-based photonic devices.

The experimental setup proposed here, schematically shown in Fig. 1, allows for various monochromatic lasers sources, as well as tunable light source as a monochromator to be applied in order to determine the refractive indices of liquid crystalline materials under tests. A critical issue in the method described here is to place a liquid crystalline material between two glass plates forming a wedge-cell of relatively small angle (of a single degree) and to introduce planar alignment of liquid crystalline molecules on glass substrates. For experimental procedure, the wedge angle has to be known and it can be determined by illuminating an empty cell with a beam perpendicular to one of the glass plates, measuring the distance between the spots coming from the reflection $\left(d_{1}\right.$ on screen $\left.\mathrm{A}\right)$.

When a liquid crystal is placed inside the wedge-cell two spots are observed on screen B due to the anisotropy of the LC material. These spots correspond to ordinary and extraodinary rays and are distanced by $d_{2 o}$ and $\mathrm{d}_{2 e}$, respectively, with respect to the initial spot given by the beam passing through the wedge-cell when it was empty. Relatively long distance $L_{2}$ between the wedge-cell and the screen B, which allows for good spatial separation of the spots, requires that the light beams used for measurements are well collimated. However, there is no need for them to be neither coherent nor polarized. The use of linearly polarized light with its direction parallel or perpendicular to the molecular orientation is not

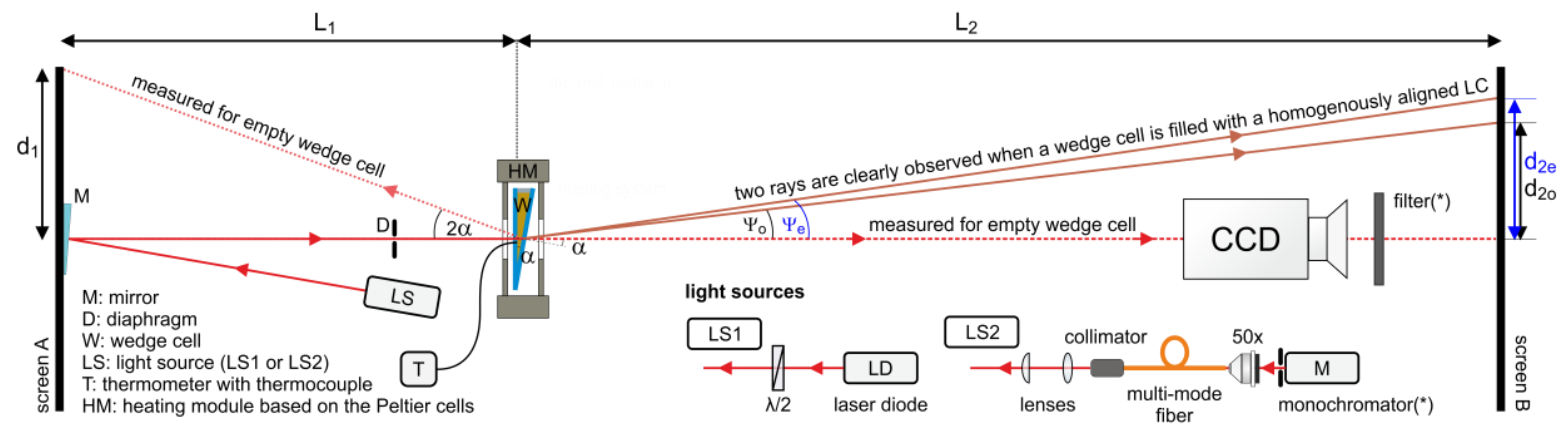

Fig. 1. Experimental setup for LC refractive index measurement with use of the wedge-cell technique. 
recommended. To obtain high precision of measurements and for automation of the measurement process, the positions of the spots on the screen $B$ are observed and registered with the use of a CCD camera. In this way, further elaboration of the photos allows for precise determination of the distances $\mathrm{d}_{20, e}$ with an accuracy less than quarter of millimeter (for laser sources) and of about millimeter when a monochromator is used (the increase mainly due to the low intensity and high spatial divergence of the beam).

Eventually, by performing the measurements of the distances between the spots on the screens $\left(d_{1}\right.$ and $\left.d_{2 o, e}\right)$ and distances between the wedge-cell and the screens $\left(L_{1}\right.$ and $L_{2}$ ), the ordinary and extraordinary refractive indices of liquid crystalline material under tests can be calculated with the use of the following formula:

(a)

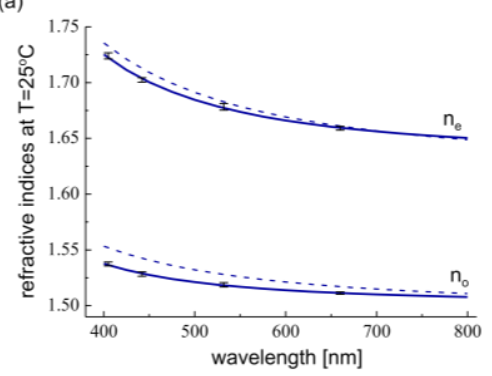

(b)

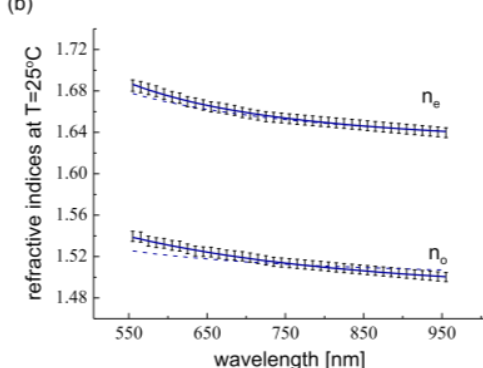

$$
\begin{aligned}
n_{o, e} & =\frac{\sin \left(\alpha+\psi_{o, e}\right)}{\sin \alpha}=\cos \psi_{o, e}\left[1+\frac{d_{2 o, e}}{L_{2} \tan \alpha}\right]= \\
& =\frac{1}{\left(L_{2}^{2}+d_{2 o, e}^{2}\right)^{1 / 2}}\left[L_{2}+\frac{d_{2 o, e}\left[L_{1}+\left(L_{1}^{2}+d_{1}^{2}\right)^{1 / 2}\right]}{d_{1}}\right],
\end{aligned}
$$

where: $\alpha$ is the wedge angle, $\psi_{o, e}$ are the angles at which ordinary and extraordinary rays are refracted at the LC-air boundary, $d_{1}$ is the distance between the spots on the screen B corresponding to the rays reflected from the glass plates in the empty wedge-cell, when one of them is perpendicular to the input light beam direction; $L_{1}$ and $L_{2}$ are the distances from the light source (mirror $\mathrm{M}$ on the screen A, Fig. 1) to the wedge-cell and from the wedgecell to the screen $\mathrm{B}$, respectively.

Fig. 2. Chromatic dispersion curves for 6CHBT LC determined at room temperature with the use of the wedge-cell method (solid lines) when four laser diodes (a) and (b) monochromator have been used as a light source. Dashed curves represent data given in the literature (slight differences are mainly due to the different temperatures in which refractive indices have been measured). (c) Thermal dependence of refractive indices for $6 \mathrm{CHBT}$, where characteristics for D-sodium-line (yellow line) are taken from the literature $[8,9]$.

Uncertentities of the refractive indices calculated with use of Eq. (1) are given by the following expression:

$$
\begin{aligned}
u\left(n_{o, e}\right) & =\frac{1}{x_{2 o, e}\left[x_{1}-L_{1}\right]} \cdot\left(\left[\frac{d_{1} d_{2 o, e}}{x_{1}}\right]^{2} u^{2}\left(L_{1}\right)+\right. \\
& +\left[\frac{\left[x_{2 o, e}^{2}-L_{2}^{2}\right]\left[x_{1}-L_{1}\right]+L_{2} d_{1} d_{2 o, e}}{x_{2 o, e}^{2}}\right]^{2} u^{2}\left(L_{2}\right)+ \\
& +\left[\frac{d_{2 o, e}\left[x_{1}^{2}-x_{1} L_{1}-d_{1}^{2}\right]}{x_{1}\left[x_{1}-L_{1}\right]}\right]^{2} u^{2}\left(d_{1}\right)+ \\
& \left.+\left[\frac{d_{1}\left[x_{2 o, e}^{2}-d_{2 o, e}^{2}\right]+L_{2} d_{2 o, e}\left[x_{1}-L_{1}\right]}{x_{2 o, e}^{2}}\right]^{2} u^{2}\left(d_{2}\right)\right)^{1 / 2},
\end{aligned}
$$

where: $x_{1}^{2} \equiv d_{1}^{2}+L_{1}^{2}, x_{2 o, e}^{2} \equiv d_{2 o, e}^{2}+L_{2}^{2}$; while $u\left(L_{\mathrm{i}}\right)$ and $u\left(d_{\mathrm{i}}\right)$ are uncertainties of the distances measured in experimental conditions and they are in the range of single millimeters for $L_{1,2}$ and a fraction of millimeters for $d_{1,2}$.

Illustrative experimental results obtained with use of described method are presented in Fig. 2. Specifically, chromatic dispersion of 6CHBT [i.e. 4-(trans-4'-nhexylcyclohexyl) isothiocyanatobenzene], a nematic LC, has been determined at room temperature $\left(\sim 25^{\circ} \mathrm{C}\right)$ for four

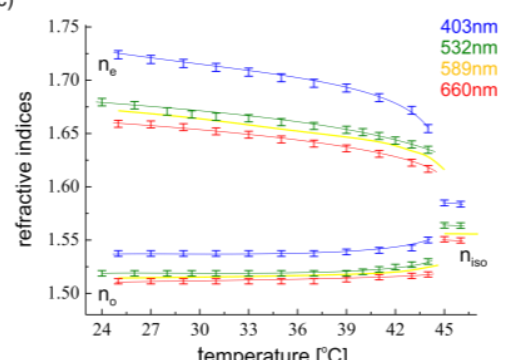

different wavelengths of the laser beams $(405 \mathrm{~nm}, 442 \mathrm{~nm}$, $532 \mathrm{~nm}$ and $660 \mathrm{~nm})$. The calculated values of refractive indices (with their uncertainties) are presented in Fig. 2a, where the dispersion curves are numerically fitted following the three-parameters Cauchy model: $n_{o, e}=A_{o, e}+$ $B_{o, e} \cdot \lambda^{-2}+C_{o, e} \cdot \lambda^{-4}$ (solid line) [10]. Analogical curves known from the literature and found with the use of different experimental methods are represented by the dashed lines and given for comparison. As already mentioned, the monochromator (here Newport MS257 $1 / 4 \mathrm{~m}$ ) can be applied, as the second, optional light source which allows for a greater number of wavelengths to be tested within a wider spectral range. The main limitations in this case are only the relatively low light intensity and high divergence of the beam leaving the monochromator output. To overcome this problem we decided to launch a light beam into the multimode fiber and then form it with the use of a collimator. Moreover, special settings of the CCD camera (i.e. high gain and long exposition time) have been applied in order to register beam spots on the screen B. Eventually, the chromatic dispersion of 6CHBT LC has been obtained for the wide spectral range (Fig. 2b) and it is also in good agreement with literature data and 
with experimental results obtained with the use of laser sources in the same setup.

It is worth noting that the proposed experimental setup is built in such a way that it allows for the thermal dependence of LC refractive indices to be characterized. For this purpose a special heating module, based on Peltier cells, is used to keep a cell thermally stabilized. Thermal dependence of 6CHBT refractive indices determined by performed measurements is presented in Fig. 2c, where the theoretical curves obtained with the use of a refractometer and a sodium lamp elsewhere [8] match well the obtained characteristics.

In conclusion, a relatively cheap and reliable technique allowing for LC chromatic dispersion determination (also as a function of temperature) has been presented. The results obtained for a typical liquid crystalline material are in good agreement with literature data. The experimental setup allows for high birefringence of liquid crystals to be measured. Specifically, the measurements performed in this setup for the 2030 LC mixture synthesized at the Military University of Technology (Warsaw) and reported elsewhere [10] concluded with a birefringence of about 0.66 and $n_{\mathrm{e}}$ of about 2.23 for a wavelength of $405 \mathrm{~nm}$ at room temperature. It is important to note that the proposed method requires a small amount of LCs to be placed under test. The accuracy of the measuring system for refractive indices is up to the third decimal. When compared to other methods, one can note some pros and cons as listed in the following table (Table 1).

Table 1. Comparison of different measurement techniques.

\begin{tabular}{|c|c|}
\hline Advantages & Disadvantages \\
\hline \multicolumn{2}{|c|}{ Wedge-cell method $[2,4]$} \\
\hline $\begin{array}{l}\checkmark \text { easy way to determine ordi- } \\
\text { nary and extraordinary refractive } \\
\text { indices of LCs (and thus birefrin- } \\
\text { gence) as a function of wave- } \\
\text { length and temperature based on } \\
\text { simple geometrical measure- } \\
\text { ments; } \\
\checkmark \text { no need to use polarized or } \\
\text { coherent beam; } \\
\checkmark \text { small amount of LC material } \\
\text { is required; } \\
\checkmark \text { measurements can be per- } \\
\text { formed for highly birefringent } \\
\text { LCs; } \\
\checkmark \text { unlimited number of light } \\
\text { sources can be applied and thus } \\
\text { wide spectral range can be con- } \\
\text { sidered. }\end{array}$ & $\begin{array}{l}\mathbf{x} \text { relatively big experimental } \\
\text { setup is required for precise } \\
\text { measurement of wedge angle and } \\
\text { for spatial separation of ordinary } \\
\text { and extraordinary rays; } \\
\mathbf{x} \text { new (empty) wedge-cells are } \\
\text { required for each monochromatic } \\
\text { laser source applied inde- } \\
\text { pendently in the measurements; }\end{array}$ \\
\hline \multicolumn{2}{|c|}{ Abbe Refractometer [7, 11] } \\
\hline $\begin{array}{l}\checkmark \text { all setup located in one device } \\
- \text { simple in use, simple measure- } \\
\text { ment procedure; } \\
\checkmark \text { compactness and portability of } \\
\text { the measurement setup; } \\
\checkmark \text { easy way for thermostat to be } \\
\text { included in the setup in order to } \\
\text { precise measurement of refractive }\end{array}$ & $\begin{array}{l}\mathbf{x} \text { measurements are mostly } \\
\text { restricted to the mean value of } \\
\text { refractive index; } \\
\mathbf{x} \text { for measurements of } n_{o} \text { and } n_{e} \\
\text { new techniques for applications } \\
\text { of orienting layers in measure- } \\
\text { ment device have to be elabo- } \\
\text { rated; }\end{array}$ \\
\hline
\end{tabular}

indices as a function of temperature (with an accuracy of $0.1^{\circ} \mathrm{C}$ );

$\checkmark$ by using filters and white light source it is possible to determine refractive indices for different wavelengths;

$\checkmark$ high precision in the order of about $10^{-4} \div 10^{-5}$ can be obtained.

\section{Fabry-Perot Etalon [1]}

$\checkmark$ possibility of $n_{o}$ and $n_{e}$ to be $\quad$ x LC cells should be coated with measured based on analysis of the interference image obtained for specific polarization;

$\checkmark$ high precision in the order of about $10^{-5} \div 10^{-6}$ can be obtained. highly reflective layers (e.g. made of gold) in order to obtain a clear interference pattern;

$\mathbf{x}$ relatively thick LC cells are required for an interference pattern to be observed;

$\mathbf{x}$ coherent light sources are required.

Speckle interferometry [3]

$\checkmark$ relatively simple optical setup for LC birefringence measurements. $\mathbf{x}$ limitations in birefringence values to be measured; $\mathbf{x}$ coherent light sources are required.

Michelson Interferometer [5-7]

$\checkmark$ even small phase shifts can be $\quad \mathbf{x}$ complicated and complex
measured;

$\checkmark$ high precision can be $\mathbf{x}$ relatively thick LC cells are achieved. $\quad$ required in order to obtain a clear interference pattern;

$\mathbf{x}$ coherent light sources are required;

$\mathbf{x}$ instability of the optical setup (e.g. air-flow) may significantly decrease the precision of refractive index determination.

This work was partially supported by the Polish National Science Center under the grant no. DEC2013/11/B/ST7/04330 (Integrated optical circuits in novel liquid crystalline structures and materials).

\section{References}

[1] M.O. Ko, S. Kim, J. Kim, B.W. Lee, M.Y. Jeon, J. Opt. Soc. Korea 19, 346 (2015).

[2] K. Thingujama, S. Sarkara, B. Choudhurya, A. Bhattacharjeea, Acta Phys. Polonica A 122(4), 754 (2012).

[3] T.A. El-Dessouki, M. Roushdy, N.I. Hendawy, M.M. Naoum, A.A. Zaki, J. Modern Physics 4, 39 (2013).

[4] J. Li, S. Wu, S. Brugioni, R. Meucci, S. Faetti, J. Appl. Phys. 97, 073501 (2005).

[5] B. Abbas, M.A. Khalil, Acta Phys. Polonica A 129, 59 (2016).

[6] S.R. Kachiraju, D. A. Gregory, Opt. Laser Techn. 44, 2361 (2012).

[7] J. Yan, M. Jiao, L. Rao, S. Wu, Opt. Expr. 18, 11450 (2010).

[8] K.A. Rutkowska, U.A. Laudyn, R.T. Rutkowski, M.A. Karpierz, T.R. Wolinski, J. Wójcik, Proc. SPIE 6582, 658215 (2007).

[9] J. Li, C. Wen, S. Gauza, R. Lu, S. Wu, J. Display Techn. 1, 51 (2005).

[10] K. Rutkowska, A. Kozak, K. Orzechowski, "Chromatic dispersion measurements of selected liquid crystalline materials for integrated optics applications," Proc. SPIE (in press) (2016).

[11] E. Nowinowski-Kruszelnicki, J. Kędzierski, Z. Raszewski, L. Jaroszewicz, R. Dąbrowski, M. Kojdecki, W. Piecek, P. Perkowski, K. Garbat, M. Olifierczuk, Opt. Appl. 42, 167 (2012). 